\title{
Comportamento de espécies florestais em plantios a pleno sol e em faixas de enriquecimento de capoeira na Amazônia
}

\author{
Cintia Rodrigues de SOUZA ${ }^{1}$, Celso Paulo de AZEVEDO², Roberval Monteiro LIMA ${ }^{3}$, Luiz Marcelo Brum ROSSI ${ }^{4}$ \\ RESUMO \\ Foram avaliadas espécies florestais nativas e exóticas em duas condiçôes de plantio: a pleno sol e em faixas de enriquecimento \\ de capoeira, a fim de gerar tecnologias para incorporação ao processo produtivo de áreas alteradas na região amazônica para \\ minimizar a pressão do desmatamento sobre a floresta natural. Os dados analisados (DAP, altura, volume por hectare e \\ sobrevivência) referem-se à idade de seis anos. No plantio a pleno sol, a espécie que apresentou o melhor desempenho foi \\ Ceiba pentandra, com médias de DAP de 19,6 cm, altura de 10,2 m e volume de $390,9 \mathrm{~m}^{3} \mathrm{ha}^{-1}$. Hymenaea courbaril também \\ apresentou crescimento satisfatório, com médias de $11,5 \mathrm{~cm}$ de DAP, $10,8 \mathrm{~m}$ de altura e $144,8 \mathrm{~m}^{3} \mathrm{ha}^{-1}$ de volume. Nos plantios \\ de enriquecimento de capoeira, a Carapa guianensis foi a espécie que apresentou o melhor desempenho, com 8,3 cm de DAP, \\ $6,4 \mathrm{~m}$ de altura e volume de $54,8 \mathrm{~m}^{3} \mathrm{ha}^{-1}$. Acacia mangium e Sclerolobium paniculatum apresentaram crescimento superior, \\ mas as taxas de sobrevivência foram muito baixas nas duas condições de plantio.
}

PALAVRAS-CHAVE: Floresta, biomassa, produção de madeira, rápido crescimento.

\section{Forest species behavior in plantations at full sun and secondary forest enrichment strips in the Amazon}

\section{ABSTRACT}

This work evaluated exotic and native forest species in two plantation conditions: full sun and secondary forest enrichment strips, for generating technologies into incorporate to the productive process of degraded lands in the Amazonian region, to minimize the deforestation pressure on the natural forest. The analyzed data ( $\mathrm{DBH}$, height, volume per hectare and survival) refers to six years old species. In the full sun plantation, the species that presented the best performance was Ceiba pentandra, with means: DBH of $19.6 \mathrm{~cm}$, height of $10.2 \mathrm{~m}$ and volume of $390.9 \mathrm{~m}^{3} \mathrm{ha}^{-1}$. Hymenaea courbaril also presented satisfactory growth, with means: DBH of $11.5 \mathrm{~cm}$, height of $10.8 \mathrm{~m}$ and volume of $144.8 \mathrm{~m}^{3} \mathrm{ha}^{-1}$. In the secondary forest enrichment plantation, Carapa guianensis was the species that presented the best performance, with means: DBH of $8.3 \mathrm{~cm}$, height of 6.4 $\mathrm{m}$ and volume of $54.8 \mathrm{~m}^{3} \mathrm{ha}^{-1}$. Acacia mangium and Sclerolobium paniculatum presented better growth. However, the survival rates were very low in both plantations.

KEYWORDS: Forest, biomass, wood production, fast-growth trees.

\footnotetext{
1 Empresa Brasileira de Pesquisa Agropecuária - Embrapa Amazônia Ocidental - E-mail: cintia.souza@cpaa.embrapa.br

2 Empresa Brasileira de Pesquisa Agropecuária - Embrapa Amazônia 0cidental - E-mail: celso.azevedo@cpaa.embrapa.br

${ }^{3}$ Empresa Brasileira de Pesquisa Agropecuária - Embrapa Amazônia Ocidental - E-mail: roberval.lima@cpaa.embrapa.br

${ }^{4}$ Empresa Brasileira de Pesquisa Agropecuária - Embrapa Amazônia Ocidental - E-mail: marcelo.rossi@cpaa.embrapa.br
} 


\section{INTRODUÇÃO}

$\mathrm{Na}$ Amazônia brasileira, cerca de 35 milhóes de hectares haviam sido desflorestados até 2007, devido à exploração desordenada e seletiva das florestas nativas (INPE, 2009). Deste total, mais de 10 milhóes de hectares encontram-se degradados ou em processo de degradação. O estabelecimento de plantios florestais em metade destas áreas representaria um incremento de $100 \%$ no total de florestas plantadas no Brasil.

$\mathrm{Na}$ Amazônia, o aproveitamento dos recursos naturais deve promover o desenvolvimento da região, mediante a geração de tecnologias para superar dois grandes desafios do seu processo de ocupaçáo: i) usar de forma sustentável os recursos naturais existentes e ii) recuperar e incorporar ao processo produtivo as áreas já alteradas.

Segundo Lentini et al. (2005), a Amazônia é a principal produtora de madeira tropical do mundo, com 25 milhóes de $\mathrm{m}^{3}$, sendo $64 \%$ consumidos no Brasil. A exploração seletiva de algumas espécies mais procuradas pelo mercado madeireiro, como, por exemplo, Virola surinamensis (ucuúba), Ceiba pentandra (sumaúma), Bertholletia excelsa (castanhado-Brasil) e Swietenia macrophylla (mogno) poderá provocar, do ponto de vista genético, a perda irreversível de muitas espécies florestais.

Para que seja revertido o atual processo de degradação da Amazônia, é necessária a adoçáo de medidas visando a ocupação racional da região e a ampliação do conhecimento científico, especificamente da pesquisa florestal e agropecuária. Dentre as alternativas para restaurar ecossistemas degradados, estáo o reflorestamento e o manejo da floresta secundária por meio do enriquecimento com espécies arbóreas de alto valor comercial (Yared, 1990; Dubois, 1992; Nepstad e Pereira, 1992; Silva e Uhl, 1992; Fernandes et al.,1993).

Vários são os benefícios ecológicos trazidos pelo reflorestamento. Entre eles estão a redução da pressão sobre as florestas nativas; absorção de $\mathrm{CO}_{2}$ da atmosfera, mitigando os efeitos do aquecimento global; regulagem do ciclo hídrico; conservação do solo; controle dos ventos; redução da poluição do ar e da água, entre outros (Victoria et al., 1991; Carpanezzi, 2000; Mora e Garcia, 2000; Franco, 1992).

As plantaçôes florestais são recomendadas para casos específicos, onde há necessidade de material mais homogêneo, para fins energéticos e onde a intenção seja introduzir espécies bem valorizadas em uma vegetaçáo de baixo valor econômico, como as capoeiras. Em todos os casos, os plantios são indicados para áreas já alteradas, onde predominem florestas secundárias e/ou áreas abandonadas.

Segundo Mendonça (1997), o enriquecimento de capoeiras com espécies úteis pode contribuir para uma fonte permanente de recursos para o pequeno produtor rural na Amazônia. Os plantios de enriquecimento devem fornecer diferentes tipos de produtos, como madeira, frutos, lenha, carvão, estacas, forragem, etc., além de outros benefícios indiretos como atração de caça, cascas, medicinais, condimentares e mel.

O objetivo deste trabalho foi avaliar o desenvolvimento de espécies florestais nativas e exóticas de uso múltiplo em dois sistemas de cultivo: plantios puros e faixas de enriquecimento de capoeira, a fim de se obter informaçóes que auxiliem a geração de tecnologias para o uso de áreas degradadas e outras atualmente excluídas do sistema de produçáo na regiáo amazônica.

\section{MATERIAL E MÉTODOS}

\section{ÁREA EXPERIMENTAL}

Os plantios localizam-se no Campo Experimental da Embrapa Amazônia Ocidental, no Km 29 da Rodovia AM 010, no Município de Manaus, Estado do Amazonas. As coordenadas são 2053'30" de latitude Sul e 59 $59^{\circ} 45^{\prime \prime}$ de longitude Oeste, com altitude média de 95 metros (Souza et al., 2003).

Segundo a classificação de Köeppen, o tipo climático é o Ami, definido por uma estaçáo menos chuvosa, porém com total pluviométrico anual elevado. A precipitação média é de $2.551 \mathrm{~mm}$ por ano e a temperatura média anual é de $25,9^{\circ} \mathrm{C}$. O solo é do tipo Latossolo Amarelo textura argilosa, com baixos valores de $\mathrm{pH}$ e CTC. As características químicas do solo, quando da realização do plantio, estão relacionadas na Tabela 1.

Tabela 1 - Características químicas do solo nas diferentes áreas experimentais.

\begin{tabular}{lccccccccc}
\hline Ensaio & $\begin{array}{c}\text { Profundidade } \\
(\mathrm{cm})\end{array}$ & $\begin{array}{c}\mathrm{pH} \\
\mathrm{H}_{2} \mathrm{O}\end{array}$ & $\begin{array}{c}\mathrm{N} \\
(\%)\end{array}$ & $\mathrm{P}$ & $\mathrm{K}$ & $\mathrm{Ca}$ & $\mathrm{Mg}$ & $\mathrm{Al}$ \\
& & & \multicolumn{3}{c}{$\mathrm{mg}_{\mathrm{dm}} \mathrm{dm}^{3}$} & \multicolumn{2}{c}{$\mathrm{cmol}_{\mathrm{c}} / \mathrm{dm}^{3}$} \\
\hline Pleno sol & $0-20$ & 4,3 & 0,17 & 1,0 & 20,0 & 0,26 & 0,11 & 1,70 \\
Capoeira & $0-20$ & 4,4 & 0,17 & 1,0 & 30,0 & 0,39 & 0,21 & 1,80 \\
\hline
\end{tabular}

\section{METODOLOGIA}

As espécies avaliadas no experimento foram selecionadas com base na rapidez de crescimento e potencialidade para uso como fonte de matéria-prima, principalmente para produção de energia e para uso em serrarias. $\mathrm{O}$ experimento foi composto por dois ensaios:

Ensaio 1: espécies florestais a pleno sol em ecossistema de terra firme.

As espécies, implantadas em 1992, foram as seguintes: Acacia mangium; andiroba (Carapa guianensis); breu sucuruba (Trattinickia burseraefolia); castanha-do-Brasil (Bertholletia excelsa); cedro (Cedrela odorata); copaíba (Copaifera multijuga); cumaru (Dipteryx odorata); ipê rosa (Tabebuia pentaphylla); jarana (Holopyxidium jarana); jatobá (Hymenaea courbaril); 
mogno (Swietenia macrophylla), sumaúma (Ceiba pentandra); taxi-branco (Sclerolobium paniculatum) e ucuúba (Virola surinamensis). $\mathrm{O}$ delineamento experimental foi em blocos ao acaso, com quatro repetiçóes. As espécies foram plantadas em parcelas de $225 \mathrm{~m}^{2}$, com 25 plantas cada, em espaçamento $3 \mathrm{~m} \times 3 \mathrm{~m}$, tendo sido avaliadas somente as nove plantas centrais.

\section{ENSAIO 2: FAIXAS DE ENRIQUECIMENTO DE CAPOEIRA.}

Instalado em janeiro de 1992, em capoeira com aproximadamente 25 anos de idade. Composto por dez espécies: Acacia mangium; andiroba (Carapa guianensis); breu sucuruba (Trattinickia burseraefolia); castanha-doBrasil (Bertholletia excelsa); cedro (Cedrela odorata); copaíba (Copaifera multijuga); cumaru (Dipteryx odorata); jatobá (Hymenaea courbaril); mogno (Swietenia macrophylla) e taxibranco (Sclerolobium paniculatum). O delineamento foi em blocos ao acaso com quatro repetiçóes. As parcelas são lineares, formadas por dez plantas por espécie, em espaçamento $3,5 \mathrm{~m}$ $\mathrm{x} 7,0 \mathrm{~m}$. As linhas de plantio foram abertas no sentido lesteoeste, com largura de $3 \mathrm{~m}$.

Em ambos os ensaios foi feita adubação na cova com aplicação de 60 gramas de superfosfato triplo por planta. Os tratos culturais consistiram de capinas e coroamento das plantas de duas a três vezes ao ano, a fim de minimizar a competição com as plantas invasoras, principalmente nos dois primeiros anos.

A freqüência de avaliaçôes dendrométricas foi semestral no primeiro ano e anual a partir deste; tendo sido avaliados os aspectos fitossanitários, sobrevivência (\%), altura $(\mathrm{H})$ e diâmetro à altura do peito (DAP), e calculados a área basal $(\mathrm{G})$, o volume ( $\mathrm{Vol})$ e os respectivos incrementos médios anuais (IMAs). A principal variável considerada neste trabalho foi o volume.
Para a coleta dos dados foi utilizado o formulário padrão do programa MIRASILV - Manejo de Informaçáo de Recursos Arbóreos, do CATIE (Centro Agronômico Tropical de Ensino e Pesquisa). Todos os dados coletados foram armazenados no formato do banco de dados MIRASILV. A análise de crescimento das espécies florestais foi realizada por meio do programa MIRASILV.

Para avaliar as diferenças entre as espécies quanto ao volume por hectare, foi utilizado o teste $\mathrm{F}$ a $95 \%$ de probabilidade e o teste de Tukey ( $95 \%$ de probabilidade) para o contraste das médias.

\section{RESULTADOS E DISCUSSÃO}

As Tabelas 2 e 3 mostram o crescimento das espécies estudadas em altura, DAP, área basal e volume, além dos respectivos incrementos médios e taxas de sobrevivência, nos ensaios pleno sol e capoeira, respectivamente. Ressalta-se que as espécies ipê rosa (T. pentaphylla); jarana (H.jarana); sumaúma (C. pentandra) e ucuúba (V. surinamensis) foram implantadas somente no ensaio a pleno sol.

O crescimento em termos de DAP, altura, área basal, volume e respectivos incrementos médios anuais e taxas de sobrevivência variaram muito entre as espécies e entre os sistemas de plantação avaliados, o que sugere a necessidade de uma seleção muito criteriosa da espécie a ser plantada em cada local.

No ensaio a pleno sol, aos seis anos de idade (Tabela 2), as espécies que obtiveram os maiores desempenhos em altura e IMA em altura foram $A$. mangium e $S$. paniculatum. Em relação ao DAP e IMA em $\mathrm{DAP}$, destacaram-se $A$. mangium e $C$. pentandra. Em seguida há um grupo de espécies com desempenho intermediário (DAPs superiores a $10 \mathrm{~cm}$ e IMAs em DAP superiores a $\left.1,8 \mathrm{~cm}^{2} o^{-1}\right): C$. odorata, $S$. paniculatum, V.surinamensis, C. guianensis, H. courbarile S. macrophylla.

Tabela 2 - Desempenho das espécies em altura (h), diâmetro à altura do peito (DAP), área basal (G), volume (VOL), sobrevivência (SOB) e respectivos incrementos médios (IMA), no ensaio "Pleno sol " aos 6 anos de idade. Manaus, 1999.

\begin{tabular}{lccccccc}
\hline ESPÉCIE & $\begin{array}{c}\mathrm{H} \\
(\mathrm{m})\end{array}$ & $\begin{array}{c}\text { DAP } \\
(\mathrm{cm})\end{array}$ & $\begin{array}{c}\text { VOL } \\
\left(\mathrm{m}^{3} \mathrm{a} \mathrm{rv}^{-1}\right)\end{array}$ & $\begin{array}{c}\text { SOB } \\
(\%)\end{array}$ & $\begin{array}{c}\text { IMAh } \\
\left(\mathrm{m} \mathrm{ano}^{-1}\right)\end{array}$ & $\begin{array}{c}\text { IMADAP } \\
\left(\mathrm{cm} \mathrm{ano}^{-1}\right)\end{array}$ & $\begin{array}{c}\text { IMAVOL } \\
\left(\mathrm{m}^{3} \mathrm{ano}^{-1}\right)\end{array}$ \\
\hline S. paniculatum & $15,1 \mathrm{a}$ & $12,1 \mathrm{bc}$ & $1,171 \mathrm{bc}$ & $63,9 \mathrm{abcd}$ & $2,5 \mathrm{a}$ & $2,0 \mathrm{bc}$ & $0,195 \mathrm{bc}$ \\
A. mangium & $14,5 \mathrm{ab}$ & $24,4 \mathrm{a}$ & $1,781 \mathrm{~b}$ & $30,6 \mathrm{~d}$ & $2,4 \mathrm{ab}$ & $4,1 \mathrm{a}$ & $0,297 \mathrm{~b}$ \\
H. courbaril & $10,8 \mathrm{bc}$ & $11,5 \mathrm{bcd}$ & $1,179 \mathrm{bc}$ & $100,0 \mathrm{a}$ & $1,8 \mathrm{bc}$ & $1,9 \mathrm{bcd}$ & $0,195 \mathrm{bc}$ \\
C. pentandra & $10,2 \mathrm{c}$ & $19,6 \mathrm{a}$ & $3,166 \mathrm{a}$ & $97,2 \mathrm{ab}$ & $1,7 \mathrm{c}$ & $3,3 \mathrm{a}$ & $0,527 \mathrm{a}$ \\
C. odorata & $9,2 \mathrm{~cd}$ & $13,7 \mathrm{~b}$ & $1,141 \mathrm{bc}$ & $77,8 \mathrm{abc}$ & $1,5 \mathrm{~cd}$ & $2,3 \mathrm{~b}$ & $0,190 \mathrm{bc}$ \\
C. multijuga & $8,8 \mathrm{~cd}$ & $8,6 \mathrm{bcd}$ & $0,393 \mathrm{c}$ & $75,0 \mathrm{abc}$ & $1,5 \mathrm{~cd}$ & $1,4 \mathrm{bcd}$ & $0,065 \mathrm{c}$ \\
D. odorata & $8,1 \mathrm{~cd}$ & $6,6 \mathrm{de}$ & $0,302 \mathrm{c}$ & $86,1 \mathrm{abc}$ & $1,4 \mathrm{~cd}$ & $1,1 \mathrm{de}$ & $0,050 \mathrm{c}$ \\
C. guianensis & $7,8 \mathrm{~cd}$ & $11,7 \mathrm{bc}$ & $0,738 \mathrm{bc}$ & $91,7 \mathrm{abc}$ & $1,3 \mathrm{~cd}$ & $1,9 \mathrm{bcd}$ & $0,123 \mathrm{bc}$ \\
S. macrophylla & $7,7 \mathrm{~cd}$ & $10,9 \mathrm{bcd}$ & $0,505 \mathrm{bc}$ & $50,0 \mathrm{~cd}$ & $1,3 \mathrm{~cd}$ & $1,8 \mathrm{bcd}$ & $0,084 \mathrm{bc}$ \\
V. surinamensis & $7,6 \mathrm{~cd}$ & $11,9 \mathrm{bcd}$ & $0,736 \mathrm{bc}$ & $86,1 \mathrm{abc}$ & $1,3 \mathrm{~cd}$ & $1,9 \mathrm{bcd}$ & $0,123 \mathrm{bc}$ \\
T. purseraefolia & $5,8 \mathrm{de}$ & $8,4 \mathrm{bc}$ & $0,206 \mathrm{c}$ & $55,6 \mathrm{bcd}$ & $0,9 \mathrm{de}$ & $1,4 \mathrm{bcd}$ & $0,034 \mathrm{c}$ \\
B. excelsa & $5,6 \mathrm{de}$ & $6,9 \mathrm{cde}$ & $0,207 \mathrm{c}$ & $50,0 \mathrm{~cd}$ & $0,9 \mathrm{de}$ & $1,5 \mathrm{cde}$ & $0,035 \mathrm{c}$ \\
T. pentaphylla & $5,0 \mathrm{de}$ & $8,1 \mathrm{~cd}$ & $0,424 \mathrm{c}$ & $83,3 \mathrm{abc}$ & $0,8 \mathrm{de}$ & $1,4 \mathrm{~cd}$ & $0,071 \mathrm{c}$ \\
H. jarana & $2,1 \mathrm{e}$ & $1,5 \mathrm{e}$ & $0,04 \mathrm{c}$ & $77,8 \mathrm{abc}$ & $0,3 \mathrm{e}$ & $0,3 \mathrm{e}$ & $0,001 \mathrm{c}$ \\
\hline
\end{tabular}

Espécies seguidas pela mesma letra não diferem estatisticamente pelo teste de Tukey a 95\% de probabilidade. 
Com relação ao volume cilíndrico, C. pentandra apresentou o melhor desempenho, muito superior às demais espécies (Tabela 2).

Com relação à sobrevivência, aos seis anos de idade, as espécies $H$. courbaril, C. guianensis e C. pentandra apresentaram os resultados mais satisfatórios (com taxas superiores a 90\%), ao contrário das espécies $A$. mangium, $S$. macrophylla e $B$. excelsa, com 31\%, 50\% e 50\%, respectivamente (Tabela 2).

Os resultados da análise de variância (ANOVA) para o plantio sob condiçóes de sombra parcial (capoeira) são mostrados na Tabela 3. As espécies que obtiveram os melhores desempenhos em altura e IMA em altura foram: $A$. mangium e $S$. paniculatum. Em relação ao DAP e IMA em DAP, as mesmas espécies destacaram-se: $A$. mangium e $S$. paniculatum. Por outro lado, estas espécies foram as que tiveram os menores índices de sobrevivência, com 50\% e 27,8\% respectivamente, superiores apenas a $S$. macrophylla, com 19,4\%. Nenhuma das espécies alcançou taxa de sobrevivência superior a $90 \%$, sendo que o melhor desempenho foi obtido pela C. guianensis (88,9\%), mostrando boa adaptação às condiçóes de iluminação parcial. Quanto ao volume cilíndrico, $A$. mangium apresentou o melhor desempenho, muito superior às demais espécies. $\mathrm{O}$ menor valor foi obtido pela $S$. macrophylla (Tabela 3).

Tabela 3 - Desempenho das espécies em altura (h), diâmetro à altura do peito (DAP), área basal (G), volume (VOL), sobrevivência (SOB) e respectivos incrementos médios (IMA), no ensaio "Capoeira" aos 6 anos de idade. Manaus, 1999.

\begin{tabular}{|c|c|c|c|c|c|c|c|}
\hline ESPÉCIE & $\begin{array}{c}\mathrm{H} \\
(\mathrm{m})\end{array}$ & $\begin{array}{l}\text { DAP } \\
\text { (cm) }\end{array}$ & $\begin{array}{c}\text { VOL } \\
\left(\mathrm{m}^{3} \text { árv }{ }^{-1}\right)\end{array}$ & $\begin{array}{l}\text { SOB } \\
(\%)\end{array}$ & $\begin{array}{l}\text { IMAh } \\
\left(\text { m ano }^{-1}\right)\end{array}$ & $\begin{array}{l}\text { IMADAP } \\
\left(\mathrm{cm} \mathrm{ano}^{-1}\right)\end{array}$ & $\begin{array}{l}\text { IMAVOL } \\
\left(\mathrm{m}^{3} \mathrm{ano}^{-1}\right)\end{array}$ \\
\hline . mangium & $\begin{array}{c}14,8 \\
a\end{array}$ & $\begin{array}{c}19,0 \\
a\end{array}$ & $1,681 \mathrm{a}$ & $\begin{array}{l}50,0 \\
b c d\end{array}$ & $2,1 \mathrm{a}$ & $3,2 \mathrm{a}$ & $0,280 \mathrm{a}$ \\
\hline S.paniculatum & $\begin{array}{c}13,5 \\
a\end{array}$ & $\begin{array}{c}14,9 \\
a\end{array}$ & $\begin{array}{c}0,568 \\
\text { bcd }\end{array}$ & $\begin{array}{c}27,8 \\
c d\end{array}$ & $2,2 \mathrm{a}$ & $2,5 \mathrm{a}$ & $\begin{array}{c}0,094 \\
\text { bcd }\end{array}$ \\
\hline C. guianensis & $\begin{array}{c}6,4 \\
b\end{array}$ & $\begin{array}{c}8,3 \\
b\end{array}$ & $\begin{array}{c}0,444 \\
\text { bcd }\end{array}$ & $\begin{array}{c}88,9 \\
a\end{array}$ & $1,1 \mathrm{~b}$ & $1,4 b$ & $\begin{array}{c}0,074 \\
\text { bcd }\end{array}$ \\
\hline C. odorata & $\begin{array}{c}5,7 \\
b\end{array}$ & $\begin{array}{l}5,6 \\
b c\end{array}$ & $\begin{array}{c}0,151 \\
\text { bcd }\end{array}$ & $\begin{array}{c}77,8 \\
a b\end{array}$ & $0,9 \mathrm{~b}$ & $0,9 b c$ & $\begin{array}{c}0,025 \\
\text { bcd }\end{array}$ \\
\hline B. excelsa & $\begin{array}{c}5,4 \\
b\end{array}$ & $\begin{array}{l}4,8 \\
b c\end{array}$ & $\begin{array}{c}0,103 \\
\text { bcd }\end{array}$ & $\begin{array}{c}72,2 \\
a b\end{array}$ & $0,9 \mathrm{~b}$ & $0,8 \mathrm{bc}$ & $\begin{array}{c}0,017 \\
\text { bcd }\end{array}$ \\
\hline C. multijuga & $\begin{array}{c}5,3 \\
b\end{array}$ & $\begin{array}{l}4,3 \\
b c\end{array}$ & $\begin{array}{c}0,073 \\
\text { bcd }\end{array}$ & $\begin{array}{c}75,0 \\
a b\end{array}$ & $0,9 \mathrm{~b}$ & $0,7 \mathrm{bc}$ & $\begin{array}{c}0,012 \\
\text { bcd }\end{array}$ \\
\hline S.macrophylla & $\begin{array}{c}3,3 \\
b\end{array}$ & $\begin{array}{l}4,5 \\
b c\end{array}$ & $0,009 d$ & $\begin{array}{c}19,4 \\
d\end{array}$ & $0,6 \mathrm{~b}$ & $0,7 \mathrm{bc}$ & $0,002 d$ \\
\hline T.burseraefolia & $\begin{array}{c}4,7 \\
b\end{array}$ & $\begin{array}{l}4,7 \\
b c\end{array}$ & $\begin{array}{c}0,058 \\
\text { bcd }\end{array}$ & $\begin{array}{l}61,1 \\
a b c\end{array}$ & $0,8 \mathrm{~b}$ & $0,8 \mathrm{bc}$ & $\begin{array}{l}0,009 \\
\text { bcd }\end{array}$ \\
\hline D. odorata & $\begin{array}{c}4,2 \\
\text { b }\end{array}$ & $\begin{array}{c}2,2 \\
C\end{array}$ & $0,023 \mathrm{~cd}$ & $\begin{array}{c}83,3 \\
a b\end{array}$ & $0,7 \mathrm{~b}$ & $0,3 \mathrm{c}$ & $\begin{array}{c}0,004 \\
\mathrm{~cd}\end{array}$ \\
\hline H. courbaril & $\begin{array}{c}4,0 \\
b\end{array}$ & $\begin{array}{c}2,7 \\
\mathrm{C}\end{array}$ & $0,024 \mathrm{~cd}$ & $\begin{array}{c}72,2 \\
a b\end{array}$ & $0,7 \mathrm{~b}$ & $0,5 \mathrm{c}$ & $\begin{array}{c}0,004 \\
\mathrm{~cd}\end{array}$ \\
\hline
\end{tabular}

Espécies seguidas pela mesma letra não diferem estatisticamente pelo teste de Tukey a $95 \%$ de probabilidade.

Entre os fatores bióticos importantes neste trabalho, se destaca o ataque da Hypsipyla grandella às espécies da família Meliaceae, independente do sistema de plantação, influenciando negativamente a sobrevivência e o crescimento durante os primeiros anos. As espécies da família Meliaceae $(S$. macrophylla, C. guianensis e C. odorata) tiveram $100 \%$ de suas árvores atacadas, causando alta mortalidade, principalmente, nas árvores de S. macrophylla. Esta praga, conhecida como broca dos ponteiros, destrói o broto terminal das árvores, diminuindo sua altura e causando o aparecimento de galhos laterais, o que reduz sensivelmente o valor comercial da madeira. C. guianensis e $C$. odorata também sofreram ataques, mas de menor intensidade, o que não afetou significativamente a sobrevivência destas espécies.

A A. mangium, apesar de ter apresentado o melhor desempenho em altura e DAP nos dois sistemas de plantio avaliados, não tem mostrado bons índices de sobrevivência à medida que aumenta a idade das plantas. Neste experimento existem parcelas reduzidas a dois indivíduos. $\mathrm{O}$ alto índice de mortalidade da espécie parece ser determinado pelo seu curto ciclo de vida, e não por características climáticas ou de nutrição do solo. Segundo CATIE (1992), esta espécie pode apresentar morte regressiva em plantaçôes após três a quatro anos de idade, especialmente em solos com alto conteúdo de alumínio. Também podem acontecer quebras de galhos e do fuste e tombamentos causados por ventos.

Comparando-se os dois sistemas de plantio, a pleno sol e em faixas de enriquecimento de capoeira, registraram-se os melhores crescimentos de todas as espécies a plena abertura, em comparação com os resultados obtidos na capoeira. Em relação à sobrevivência, no geral as espécies apresentaram maiores valores também no plantio a pleno sol, com exceção das seguintes, que se adaptaram melhor às condiçôes de sombreamento: $A$. mangium, $T$. burseraefolia e $B$. excelsa. $\mathrm{O}$ fato da $A$. mangium ter apresentado maior taxa de sobrevivência no plantio sombreado pode ser devido ao fato de que neste sistema as árvores ficaram mais protegidas dos ventos, o que diminuiu as quedas e tombamentos e, conseqüentemente, a mortalidade (Tabela 4).

Considerando o volume, que é o principal parâmetro para avaliação das espécies neste trabalho, e também a sobrevivência, a espécie que obteve o melhor desempenho na condição de pleno sol foi $C$. pentandra, espécie amazônica de alto valor comercial utilizada na fabricação de compensados, laminados, embarcaçóes, caixas e brinquedos, com volume cilíndrico de 390,86 $\mathrm{m}^{3} \mathrm{ha}^{-1}$ e sobrevivência de $97,2 \%$. Esta pode ser considerada uma das espécies nativas mais promissoras para plantios comerciais na região amazônica. Em seguida vem $H$. courbaril, espécie nativa cuja madeira, muito dura, é empregada na construção civil e na fabricação de móveis e esquadrias, com volume cilíndrico de 144,81 $\mathrm{m}^{3}$ ha $^{-1}$ e sobrevivência de $100 \%$. A $A$. mangium, espécie australiana utilizada principalmente na produção de energia, obteve crescimento superior a esta última, mas neste sistema de plantio foi a espécie que apresentou o índice de sobrevivência 
Tabela 4 - Comparação do desempenho das espécies em altura (h), diâmetro à altura do peito (DAP), área basal (G), volume (VOL), sobrevivência (SOB) e respectivos incrementos médios (IMA) das espécies, no ensaios "Pleno Sol" e "Capoeira" aos 6 anos de idade. CPAA/EMBRAPA, Manaus, 1999.

\begin{tabular}{|c|c|c|c|c|c|}
\hline ESPÉCIE & Ambiente & $\begin{array}{c}\mathrm{H} \\
(\mathrm{m})\end{array}$ & $\begin{array}{l}\text { DAP } \\
(\mathrm{cm})\end{array}$ & $\begin{array}{c}\text { VOL } \\
\left(m^{3} \mathrm{ha}^{-1}\right)\end{array}$ & $\begin{array}{l}\text { SOB } \\
(\%)\end{array}$ \\
\hline \multirow{2}{*}{ S. paniculatum } & Pleno Sol & 15,13 & 12,05 & 144,57 & 63,9 \\
\hline & Capoeira & 13,45 & 14,91 & 70,12 & 27,8 \\
\hline \multirow{2}{*}{ A. mangium } & Pleno Sol & 14,53 & 24,36 & 219,87 & 30,6 \\
\hline & Capoeira & 14,84 & 19,02 & 207,41 & 50,0 \\
\hline \multirow{2}{*}{ H. courbaril } & Pleno Sol & 10,76 & 11,47 & 144,81 & 100,0 \\
\hline & Capoeira & 3,99 & 2,74 & 2,96 & 72,2 \\
\hline \multirow{2}{*}{ C. odorata } & Pleno Sol & 9,20 & 13,74 & 140,86 & 77,8 \\
\hline & Capoeira & 5,66 & 5,55 & 18,64 & 77,8 \\
\hline \multirow{2}{*}{ C. multijuga } & Pleno Sol & 8,82 & 8,61 & 48,39 & 75,0 \\
\hline & Capoeira & 5,26 & 4,28 & 9,01 & 75,0 \\
\hline \multirow{2}{*}{ D. odorata } & Pleno Sol & 8,14 & 6,59 & 37,28 & 86,1 \\
\hline & Capoeira & 4,20 & 2,22 & 2,84 & 83,3 \\
\hline \multirow{2}{*}{ C. guianensis } & Pleno Sol & 7,85 & 11,67 & 91,11 & 91,7 \\
\hline & Capoeira & 6,43 & 8,28 & 54,81 & 88,9 \\
\hline \multirow{2}{*}{ S. macrophylla } & Pleno Sol & 7,71 & 10,87 & 62,47 & 50,0 \\
\hline & Capoeira & 3,34 & 4,47 & 1,11 & 19,4 \\
\hline \multirow{2}{*}{ T. burseraefolia } & Pleno Sol & 5,83 & 8,37 & 25,43 & 55,6 \\
\hline & Capoeira & 4,72 & 4,69 & 7,16 & 61,1 \\
\hline \multirow{2}{*}{ B. excelsa } & Pleno Sol & 5,59 & 6,90 & 25,68 & 50,0 \\
\hline & Capoeira & 5,41 & 4,81 & 12,72 & 72,2 \\
\hline
\end{tabular}

mais baixo, com apenas $30,6 \%$ de indivíduos vivos aos seis anos de idade.

$\mathrm{Na}$ capoeira, a espécie que apresentou o melhor desempenho em volume e sobrevivência aos seis anos de idade foi $C$. guianensis, espécie de uso múltiplo (sua madeira é muito valorizada pela indústria, e produz também o óleo de suas sementes, que é insetífugo e medicinal, bastante utilizado no Norte do País), com $54,81 \mathrm{~m}^{3} \mathrm{ha}^{-1}$ de volume cilíndrico e $88,9 \%$ de sobrevivência. $A$. mangium e $S$. paniculatum (espécie cuja madeira é de boa qualidade para produção de lenha e carvão, além de ser muito utilizada na recuperação de áreas alteradas) apresentaram melhores desempenhos em volume, porém, suas taxas de sobrevivência não foram satisfatórias (50\% e $27,8 \%$, respectivamente), o que desencoraja seu plantio nessas condiçóes.

Outros estudos corroboram estes resultados. No Acre, Oliveira et al. (1996), avaliando plantio de C. pentandra em solo podzólico vermelho-escuro, obtiveram médias de altura de $1,67 \mathrm{~m}$ e diâmetro do colo de $4,89 \mathrm{~cm}$, aos 12 meses de idade.

Em plantios visando o enriquecimento da regeneraçáo natural, também no Acre, a espécie apresentou, aos 5 anos, altura média de 3,1 m e DAP de 2,23 cm. Entretanto, após o segundo ano de plantio, a espécie apresentou alta mortalidade, chegando a $50 \%$ aos cinco anos, provavelmente devido ao fechamento do dossel (d'Oliveira, 2000).
Chinea-Rivera (1990), apud Neves (1999), relata que a espécie apresentou aos quatro anos incremento médio anual (IMA) em altura de 1,6 m e em DAP de 3,2 cm, em plantios na Costa Rica. Após desbastes seletivos, o incremento corrente anual (ICA) em altura foi superior a $2 \mathrm{~m}$.

Em experimento conduzido em Manaus, a C. pentandra plantada em espaçamento de $3 \times 3 \mathrm{~m}$, em latossolo amarelo de baixa fertilidade, apresentou aos quatro anos altura de $8,58 \mathrm{~m}$, diâmetro de $17,21 \mathrm{~cm}$ e sobrevivência de $97,2 \%$ (Neves, 1999). O mesmo autor, em estudo comparando o desenvolvimento silvicultural da $C$. pentandra e da Virola surinamensis, concluiu que a primeira apresentou os maiores valores de altura, DAP e incremento médio em volume, aos 55 meses de idade (10,6 $\mathrm{m}$ de altura, 24,7 $\mathrm{cm}$ de DAP e 0,54 $\mathrm{m}^{3} \mathrm{ha}^{-1}$ ano $^{-1}$ contra $5,4 \mathrm{~m}$ de altura, $8,3 \mathrm{~cm}$ de DAP e 0,03 $\left.\mathrm{m}^{3} \mathrm{ha}^{-1} \mathrm{ano}^{-1}\right)$.

Caliri et al. (2000) compararam o crescimento da $C$. pentandra plantada em várzea e em terra-firme no Amazonas, em plantios puros e como componente de sistemas agroflorestais (consórcios de espécies frutíferas e florestais). O melhor desempenho foi conseguido no SAF, com valores médios de DAP de $44 \mathrm{~cm}$ e altura de $14,2 \mathrm{~m}$, aos cinco anos de idade. Isso foi devido ao fato de que neste sistema as plantas receberam adubação, o que não aconteceu nos outros ambientes testados. Não foi possível determinar até que ponto a interação entre as diversas espécies também contribuiu para o desenvolvimento da espécie. O segundo melhor desempenho foi obtido na várzea, com médias de 29,7 cm de DAP e 12,2 $\mathrm{m}$ de altura, aos sete anos de idade, devido aos depósitos de nutrientes que ocorrem anualmente com as enchentes do rio Solimóes. O desempenho mais baixo ocorreu em terra-firme, com valores médios de DAP de $19,7 \mathrm{~cm}$ e altura de 9,7 m, aos sete anos de idade.

Souza et al. (2003) testaram o desempenho de 25 espécies florestais nativas e exóticas em uma área degradada localizada no Campo Experimental da Embrapa Amazônia Ocidental, no Município de Manaus/AM, em espaçamento $3 \mathrm{~m} \mathrm{x} 4 \mathrm{~m}$. A sumaúma apresentou as seguintes características dendrométricas, aos quatro anos de idade: $8,9 \mathrm{~cm}$ de DAP, 4,1 $\mathrm{m}$ de altura e $10,55 \mathrm{~m}^{3} \mathrm{ha}^{-1}$ ano $^{-1}$. Apesar do baixo desempenho da espécie, seu crescimento foi equivalente a outras como andiroba (Carapa guianensis), eucalipto (Eucalyptus grandis x E. urophylla), freijó (Cordia sp.) e ucuúba (Virola surinamensis). Tais resultados mostram-se inferiores aos de outros experimentos devido principalmente ao solo pobre da área experimental, confirmando que a espécie é extremamente exigente no que diz respeito à fertilidade do solo.

Já $H$. courbaril, em plantios florestais na região de Belterra (PA), apresentou sobrevivência de 56\%, DAP médio de 4,5 $\mathrm{cm}$ e altura média de $2,8 \mathrm{~m}$ em plantios a pleno sol, aos seis 
anos e meio de idade; sob sombreamento, a sobrevivência foi de $87 \%$ aos 15 anos de idade (Carvalho, 1998).

Para C. guianensis, Kanashiro e Yared (1991) relataram incrementos em DAP variando entre $1,34 \mathrm{~cm}^{-1} \mathrm{ano}^{-1}$ (sombra parcial) e $1,85 \mathrm{~cm} \mathrm{ano}^{-1}$ (pleno sol), aos sete anos. Em plantios de 19 anos de idade em Manaus, a espécie apresentou sobrevivência de $91 \%$, DAP de 23,9 cm e altura de 15,3 $\mathrm{m}$, superando o desempenho das demais espécies estudadas. Entretanto, sua altura comercial foi de apenas 4,5 m, devido ao ataque da $H$. grandella. Na Reserva Ducke, também em Manaus, C. guianensis desenvolveu-se bem em pleno sol, apresentando sobrevivência de $98 \%$ com nove anos de idade, 6,24 m de altura e 7,3 cm de DAP (Loureiro et al., 1979).

Neves et al. (1993) avaliaram o comportamento de espécies florestais plantadas a pleno sol em Manaus/AM, aos 12 meses de idade. As espécies que apresentaram melhor desempenho quanto à altura e ao DAP foram: C. guianensis (com 1,65 m de altura e 3,4 cm de DAP), C. odorata $(2,55 \mathrm{~m}$ de altura e $6,2 \mathrm{~cm}$ de DAP), S. macrophylla $(2,17 \mathrm{~m}$ de altura e $3,8 \mathrm{~cm}$ de DAP), Acacia mangium (1,65 $\mathrm{m}$ de altura e 3,4 $\mathrm{cm}$ de DAP) e T. burseraefolia (1,68 $\mathrm{m}$ de altura e 2,4 $\mathrm{cm}$ de DAP). Todas as espécies apresentaram sobrevivência de $72 \%$ a $100 \%$.

Mendonça (1997) testou 32 leguminosas arbóreas para a identificação de espécies de múltiplo uso com potencial para enriquecimento lenhoso de capoeiras abandonadas na Amazônia. Os plantios foram efetuados em dois sistemas de cultivo: em plena abertura e sob semi-sombreamento, sem aplicação de fertilizantes. No cultivo a pleno sol, as espécies D. odorata, Senna siamea e Swartzia ployphylla adaptaramse ao ambiente estudado e podem ser recomendadas em novos estudos para recuperação de áreas degradadas. Apuleia leiocarpa, Caesalpinia ferrea, Calliandra calothyrsus, $H$. courbaril e Swartzia sericea apresentaram baixo desempenho e pouca adaptação ao ambiente. No enriquecimento de capoeira, A. leiocarpa e Stryphnodendron guianense destacaramse como de rápido crescimento, enquanto Anadenanthera peregrina, Caesalpinia echinata, C. ferrea, Clathrotropis nitida e Enterolobium schomburgkii tiveram baixo desempenho.

Observando-se o desempenho em sobrevivência e produção volumétrica das espécies estudadas a pleno sol e em faixas de enriquecimento de capoeira, pode-se concluir que, para plantios a pleno sol na região amazônica, nas condiçóes avaliadas, a melhor espécie é a $C$. pentandra (com finalidade de produção de madeira para painéis compensados). A $A$. mangium, apesar de ter apresentado melhor desempenho, não apresenta bons índices de sobrevivência à medida que aumenta a idade das plantas. No caso de plantios de enriquecimento de capoeira, a $C$. guianensis apresentou o melhor desempenho, podendo ser utilizada para fins de produção madeireira ou de óleo medicinal.

\section{AGRADECIMENTOS}

Ao técnico da Embrapa Amazônia Ocidental Moacyr J. A. Sarrazin, pelas coletas de dados e auxílio nas atividades de campo.

\section{BIBLIOGRAFIA CITADA}

Caliri, G. J. A. et al. 2000. Caracterização do crescimento da sumaúma (Ceiba pentandra) sob diversas condições de plantio na Amazônia Central, p. 78-81. In: Congresso Brasileiro de Sistemas Agroflorestais, 3., 2000, Manaus. Sistemas agroflorestais: manejando a biodiversidade e compondo a paisagem rural resumos expandidos. Embrapa Amazônia Ocidental. Manaus.

Carpanezzi, A. A. 2000. Benefícios indiretos da floresta, p. 19-56. In: Galvão, A. P. M. (Org.). Reflorestamento de propriedades rurais para fins produtivos e ambientais: um guia para açôes municipais e regionais. Embrapa Comunicação para Transferência de Tecnologia; Colombo: Embrapa Florestas., Brasília, DF.

Carvalho, J. O. P. de. 1998. Silvicultura de cinco espécies arbóreas da Amazônia: indicaçôes de usos de seus produtos madeireiros e nãomadeireiros. EMBRAPA-CPATU. 3 p. (EMBRAPA-CPATU. Comunicado Técnico, 90), Belém, PA.

CATIE. 1992. Mangium (Acacia mangium Willd): especie de árbol de uso múltiple en América Central. Turrialba, Colección de Guias Silviculturales $56 \mathrm{pp}$.

d'Oliveira, M. V. N. 2000. Artificial regeneration in gaps and skidding trails after mechanised forest exploitation in Acre, Brazil. Forest Ecology and Management, 127: 67-76.

Dubois, J. C. L. 1992. Sistemas agroflorestais: chave para o desenvolvimento da Amazônia. REBRAF, Rio de Janeiro: 15 pp.

Fernandes, E. C. M.; Neves, E. J. M.; Matos, J. C. de S. 1993. Agrofloresta, capoeiras manejadas e plantaçôes florestais para a reabilitação de áreas desmatadas na Amazônia Brasileira, p. 96-101. In: Congresso Florestal Panamericano, 1.; Congresso Florestal Brasileiro, 7., Curitiba. Floresta para o desenvolvimento: política, ambiente, tecnologia e mercado: Anais...SBS; [S.1.]: SBEF, Vol. 3. São Paulo.

Franco, M. A. G. 1992. Características climáticas das áreas destinadas a atividades de reflorestamento, p. 17-24. In: Novaes, A. B. et al. Reflorestamento no Brasil. Vitória da Conquista: Universidade Estadual do Sudoeste da Bahia.

INPE. Monitoramento da floresta Amazônica brasileira por satélite - projeto PRODES. Disponível em: (http://www.obt.inpe.br/ prodes/) Acesso em: 16/06/2009.

Kanashiro, M.; Yared, J. A. G. 1991. Experiências com plantios florestais na Bacia Amazônica, p. 117-137. In: Simpósio Internacional “O Desafio Das Florestas Neotropicais”, Curitiba: Universidade Federal do Paraná; Freiburg: Universidad Albert Ludwig.

Lentini, M. et al. 2005. Fatos florestais da Amazônia 2005. Belém, PA: IMAZON, $142 \mathrm{pp}$.

Loureiro, A. A.; Silva, M. F da; Alencar, J. da C. 1979. Essências madeireiras da Amazônia. Instituto Nacional de Pesquisas da Amazônia, INPA, Vol. 2. Manaus. 
Mendonça, M. A. de F. 1997. Seleção de leguminosas arbóreas para plantios de enriquecimento florestal: germinação das sementes $e$ acompanhamento do crescimento após a introdução em capoeira em solo podzólico vermelho-amarelo. Dissertaçáo de Mestrado, Universidade Federal do Amazonas, Manaus, Amazonas. 58 pp.

Mora, A. L.; Garcia, C. H. 2000. A cultura do eucalipto no Brasil. São Paulo: SBS. 112 pp.

Nepstad, D.; Pereira, C. 1992. Conceitualização: extensão e contextualização de áreas alteradas/degradadas da Região Amazônica. Belém, DF: Woods Hole Research Center: EMBRAPA-CPATU, Trabalho apresentado no Seminário sobre Recuperação de áreas degradadas. 9 pp.

Neves, E. J. M. et al. 1993. Comportamento de espécies florestais a pleno sol e em linhas de enriquecimento em Manaus-AM, 756 pp. In: Congresso Florestal Panamericano, 1.; Congresso Florestal Brasileiro, 7. Curitiba. Floresta para o desenvolvimento: política, ambiente, tecnologia e mercado: Anais...SBS; [S.l.]: SBEF, Vol. 2, São Paulo.

Neves, E. J. M. 1999. Biomassa e acúmulo de nutrientes nos diferentes compartimentos de Ceiba pentandra (L.) Gaertn e Virola surinamensis (Rol.) Warb plantadas na Amazônia Ocidental Brasileira. Tese de Doutorado, Universidade Federal do Paraná, Curitiba, Paraná. 189 pp.

Oliveira, L. C. de et al. 1996. Comportamento silvicultural e custos de implantação de sumaúma (Ceiba pentandra (L.) Gaertn) em diferentes espaçamentos. (Embrapa Acre. Pesquisa em andamento, n. 88). Rio Branco: Embrapa Acre. 3 pp.
Silva, J. N. M.; Uhl, C. 1992. Atividade madeireira como uma alternativa viável para a utilizaçáo sustentada dos recursos florestais na Amazônia brasileira, p. 257-260. In: Seminário Internacional sobre Meio Ambiente, Pobreza e Desenvolvimento da Amazônia - Sindamazônia, Anais...SECTAMA: PRODEPA, Belém, PA.

Souza, C. R. de, Rossi, L. M. B.; Azevedo, C. P. de; Lima, R. M. B. de. 2003. Desempenho de espécies florestais potenciais para plantios na Amazônia Central. In: congresso florestal brasileiro, 8. São Paulo. Benefícios, produtos e serviços da floresta: oportunidades $e$ desafios do século XXI. São Paulo: Sociedade Brasileira de Silvicultura: Sociedade Brasileira de Engenheiros Florestais. 1 CD-ROM.

Victoria, R. L. et al. 1991. A Amazônia brasileira e seu papel no aumento da concentração de $\mathrm{CO}_{2}$ na atmosfera, p. 9-20. In: Val, A. L.; Figlioulo, R.; Feldberg, E. (Ed.). Bases cientificas para estratégias de preservação e desenvolvimento da Amazônia: fatos e perspectivas. INPA, Manaus.

Yared, J. A. G. 1990. Silvicultura de algumas espécies nativas da Amazônia, p. 119-121. In: CONGRESSO FLORESTAL BRASILEIRO, 6. Campos do Jordão. Florestas e meio ambiente: conservação e produção, patrimônio social. Anais... Sociedade Brasileira de Silvicultura: Sociedade Brasileira de Engenheiros Florestais. Vol. 3. Campos do Jordão.

Recebido em:23/11/2007

Aceito em: 15/07/2009 
